Bài báo khoa học

\title{
Nghiên cứu ứng dụng thuật toán nhận dạng cấu trúc bảng dựa trên phát hiện đối tượng
}

\author{
Ngô Đại Dương ${ }^{1}$, Hải Hồng Phan ${ }^{\text {1*, Phạm Lê Phương }}{ }^{2}$ \\ ${ }^{1}$ Học Viện Kỹ thuật Quân Sự; hongpth@lqdtu.edu.vn; daiduong28789@hotmail.com \\ ${ }^{2}$ Trung tâm Thông tin và Dữ liệu KTTV; phuongp180@gmail.com \\ *Tác giả liên hệ: hongpth@lqdtu.edu.vn; Tel: +84-372576968
}

Ban Biên tập nhận bài: 05/3/2021; Ngày phản biện xong: 10/5/2021; Ngày đăng bài: 25/7/2021

Tóm tắt: Nhận dạng cấu trúc bảng là vấn đề quan trọng trong số hóa tài liệu. Với sự phát triển của các kỹ thuật học sâu hiện nay việc phát hiện bảng đã có nhiều bước tiến lớn, trong khi đó nhận dạng cấu trúc bảng vẫn gặp rất nhiều khó khăn do cấu trúc bảng phức tạp, đặc biệt với dữ liệu thực tế. Bài báo này đề xuất một phương pháp ứng dụng mô hình Cascade mask $\mathrm{R}-\mathrm{CNN}$ x $101 \mathrm{FPN}$ deconv để nhận dạng hàng và cột. Bài báo cũng đề xuất sử dụng mô hình Faster R-CNN để nhận dạng các ô trong bảng, từ đó đưa ra cấu trúc bảng. Thuật toán đề xuất được đánh giá trên tập dữ liệu phổ biến như TabStructDB và các tài liệu thu thập được từ các trạm Khí tượng thủy văn. Kết quả thực nghiệm đạt $90 \%$ độ chính xác trên các tập dữ liệu này. Thuật toán có khả năng áp dụng hiệu quả vào việc nhận dạng cấu trúc bảng của các tài liệu thông thường; đặc biệt thuật toán có khả năng xử lý với các tài liệu lịch sử và các chữ viết tay, phù hợp với đặc điểm tài liệu của ngành Khí tượng thủy văn. Từ đó góp phần vào việc số hóa tài liệu, lưu trữ và truy xuất thông tin dữ liệu của ngành Khí tượng thủy văn.

Từ khóa: Nhận dạng cấu trúc bảng; Nhận dạng ô; Khí tượng thủy văn.

\section{Mở đầu}

Hiện nay, chuyển đổi số là một trong những mục tiêu phát triển của quốc gia vì lợi ích mà nó mang lại cho sự phát triển kinh tế, xã hội. Một trong các nhiệm vụ quan trọng của chuyển đổi số là số hóa tài liệu. Các tài liệu sổ sách được scan hoặc chụp ảnh rồi lưu vào máy tính dưới dạng định dạng ảnh. Tuy nhiên, để khai thác dữ liệu hiệu quả hơn cần số hóa các file này thành các dạng văn bản. Tài liệu được chia thành nhiều vùng như: vùng đoạn văn bản, vùng tiêu đề, vùng ảnh, vùng bảng...Trong đó, vùng bảng thường chứa nhiều thông tin, nhất là với các tài liệu sổ sách, kỹ thuật. Do đó, trích rút thông tin bảng trong tài liệu là một khâu quan trọng, quyết định trong số hóa tài liệu.

Cấu trúc bảng trong tài liệu rất đa dạng với nhiều kích thước khác nhau và nhiều loại khác nhau (như bảng có viền, bảng ít viền, bảng không viền). Ngoài ra bảng có thể từ các tài liệu mới, cũ khác nhau và có nhiều loại cấu trúc rất phức tạp. Nhận dạng cấu trúc bảng là xác định các ô (cell), hàng, cột và mối quan hệ phân cấp giữa các ô. Đây là một bài toán rất phức tạp và hiện nay vẫn chưa có một giải pháp tổng thể nào giải quyết được toàn bộ các dữ liệu bảng trong thực tế và kết quả nhận dạng chưa cao.

Một số nghiên cứu tiêu biểu về lĩnh vực này có thể kể tới như: Năm 1997, nghiên cứu [1] lần đầu đề xuất một phương pháp trích rút bảng dựa trên cấu trúc dữ liệu được gọi là đồ thị căn chỉnh ký tự (CAG-Charater Alignment Graph), một đồ thị căn chỉnh ký tự được hình thành bằng cách kiểm tra sự liên kết khoảng trắng trong các khối văn bản liền kề. Một số 
tham số khác như lỗ (số khoảng trống giữa các cột) và khoảng trống (số khoảng trống liền kề như vậy trên mỗi dòng) được sử dụng để kiểm tra cấu trúc khoảng trống trong một khối dữ liệu. Cùng với sự phát triển của các kỹ thuật học sâu, năm 2017, nghiên cứu [2] đã đề xuất một hệ thống từ đầu đến cuối để hiểu bảng trong tài liệu được gọi là DeepDeSRT. Đầu tiên, nghiên cứu sử dụng mô hình mạng Faster-RCNN [3] cho nhiệm vụ phát hiện bảng, tiếp theo sử dụng mô hình phân đoạn ngữ nghĩa FCN [4] thực hiện nhiệm vụ nhận dạng hàng và cột ở trong bảng. Nghiên cứu thực hiện huấn luyện trên tập dữ liệu Marmot [5], và đánh giá trên tập dữ liệu ICDAR 2013 [6]. Kết quả đạt được $\mathrm{F}_{1}$ là $96,67 \%$ cho nhiệm vụ phát hiện bảng và $91,44 \%$ cho nhiệm vụ nhận dạng cấu trúc bảng. Năm 2019, nghiên cứu [7] đã đề xuất một mô hình học sâu tại hội nghị quốc tế về phân tích và nhận dạng tài liệu (ICDAR) dựa vào các phép tích chập biến dạng để nhận dạng hàng và cột của bảng, từ đó có thể xác định các ô và cấu trúc trong bảng.

Nghiên cứu [8] đã đề xuất một mô hình học sâu CascadeTabNet sử dụng để phát hiện và nhận dạng cấu trúc bảng từ đầu đển cuối. Các thử nghiệm của nghiên cứu được thực hiện trên các tập dữ liệu ICDAR 2013, ICDAR 2019 [9], Tablebank [10].

Các phương pháp kể trên phần lớn giải quyết trên ảnh tài liệu xây dựng cho các cuộc thi, chưa xử lý và chứng minh tính hiệu quả với dữ liệu thực tế. Hai là, các dữ liệu thường có một loại lịch sử hoặc hiện đại. Trong khi dữ liệu cần số hóa thực tế có thể gồm cả hai. Dữ liệu của các trạm Khí tượng thủy văn là một trong các dữ liệu như vậy, nó bao gồm cả các tài liệu mới và cũ. Bài báo này đề xuất một phương pháp dựa trên các kỹ thuật phát hiện đối tượng theo hướng học sâu để giải quyết bài toán thực tế. Phương pháp đề xuất được đánh giá trên tập dữ liệu phổ biến TabStructDB mở rộng [1] cho nhiệm vụ nhận dạng hàng và cột, đánh giá nhận dạng các ô trong cột trên tập dữ liệu DetectCell tự xây dựng và tập dữ liệu Khí tượng thủy văn.

Mục đích của nghiên cứu nhằm: (1) đề xuất thuật toán nhận dạng cấu trúc bảng dựa trên mô hình Cascade mask $\mathrm{R}-\mathrm{CNN}$ x101FPN deconv cho nhiệm vụ nhận dạng hàng và cột và Faster R-CNN cho nhiệm vụ nhận dạng các ô từ các cột ở trong bảng; (2) phương pháp đề xuất được chứng minh hiệu quả trên các tập dữ liệu, đặc biệt dữ liệu thực tế là các tài liệu Khí tượng thủy văn.

\section{Phương pháp nghiên cứu}

\subsection{Dũ liệu nghiên cứu}

\subsubsection{Tập dữ liệu TabStructDB mở rộng và DetectCell}

Với nhiệm vụ nhận dạng cấu trúc bảng, bài báo sử dụng dữ liệu TabStructDB mở rộng để tăng cường dữ liệu. Dữ liệu này được gán nhãn cho từng hàng và từng cột của bảng. Tổng cộng bao gồm có 2079 ảnh (ban đầu có 1081 ảnh) trong đó có tăng cường thêm 868 ảnh từ tập dữ liệu Tablebank [10] và 130 ảnh của tập dữ liệu quan trắc KTTV. Dữ liệu này được sử dụng để nhận dạng hàng và cột cho bảng. Với nhiệm vụ nhận dạng ô (cell), bài báo đề xuất tập dữ liệu DetectCell với 1.172 ảnh. Mỗi ảnh gán nhãn cho từng ô, dữ liệu gồm cả tập dữ liệu mở rộng và tập dữ liệu quan trắc KTTV.

\subsubsection{Tập dữ liệu quan trắc KTTV}

Tập dữ liệu Khí tượng thủy văn thu thập bao gồm các sổ sách, bảng biểu từ nhiều năm trước, nó bao gồm cả dữ liệu hiện đại và lịch sử. Có 13 loại sổ sách kỹ thuật khác nhau: sổ quan trắc khí tượng cơ bản, sổ quan trắc mực nước, sổ dùng khi đo độ sâu, tổng bức xạ giờ, sổ quan trắc bốc hơi GGI-3000..., mỗi loại sổ sách thường có các bảng thông thường và các bảng đặc trưng cho từng loại sô. 


\begin{tabular}{|c|c|c|c|c|c|c|c|c|c|}
\hline \multirow[t]{3}{*}{$\mathrm{n}$} & \multirow[t]{2}{*}{ Meson } & \multirow[t]{2}{*}{$L$} & \multirow[t]{2}{*}{$S$} & \multirow[t]{2}{*}{$\mathrm{J}$} & \multirow[t]{2}{*}{$J^{P C}$} & \multirow{2}{*}{$\begin{array}{l}\text { our calculated } \\
\sqrt{\left\langle r^{2}\right\rangle}\end{array}$} & \multirow{2}{*}{$\begin{array}{c}\text { Theor. } \sqrt{\left\langle r^{2}\right\rangle} \\
\text { with } \\
\text { potential model [?] }\end{array}$} & \multirow[t]{2}{*}{$|R(0)|^{2}$} & Ngurái alo \\
\hline & & & & & & & & & \\
\hline & & & & & & $\mathrm{fm}$ & $\mathrm{fm}$ & $\mathrm{GeV}^{3}$ & \\
\hline \multirow[t]{4}{*}{$2 \mathrm{D}$} & $\eta_{c 2}\left(2^{1} D_{2}\right)$ & 2 & 0 & 2 & $2^{-+}$ & 1.2595 & - & $\approx 0$ & \\
\hline & $\psi\left(2^{3} D_{1}\right)$ & 2 & 1 & 1 & $1^{--}$ & 1.2112 & - & $\approx 0$ & \\
\hline & $\psi_{2}\left(2^{3} D_{2}\right)$ & 2 & 1 & 2 & $2^{--}$ & 1.2556 & - & $\approx 0$ & \\
\hline & $\psi_{3}\left(2^{3} D_{3}\right)$ & 2 & 1 & 3 & $3^{--}$ & 1.2793 & - & $\approx 0$ & \\
\hline \multirow[t]{4}{*}{$3 \mathrm{D}$} & $\eta_{c 2}\left(3^{1} D_{2}\right)$ & 2 & 0 & 2 & $2^{-+}$ & 1.57397 & - & $\approx 0$ & \\
\hline & $\psi\left(3^{3} D_{1}\right)$ & 2 & 1 & 1 & $1^{--}$ & 1.52397 & - & $\approx 0$ & \\
\hline & $\psi_{2}\left(3^{3} D_{2}\right)$ & 2 & 1 & 2 & $2^{--}$ & 1.5706 & - & $\approx 0$ & \\
\hline & $\psi_{3}\left(3^{3} D_{3}\right)$ & 2 & 1 & 3 & $3^{--}$ & 1.59398 & - & $\approx 0$ & \\
\hline \multirow[t]{4}{*}{$4 \mathrm{D}$} & $\eta_{c 2}\left(4^{1} D_{2}\right)$ & 2 & 0 & 2 & $2^{-+}$ & 1.8596 & $=$ & $\approx 0$ & \\
\hline & $\psi\left(4^{3} D_{1}\right)$ & 2 & 1 & 1 & $1^{--}$ & 1.80785 & - & $\approx 0$ & \\
\hline & $\psi_{2}\left(4^{3} D_{2}\right)$ & 2 & 1 & 2 & $2^{--}$ & 1.8565 & - & $\approx 0$ & \\
\hline & $\psi_{3}\left(4^{3} D_{3}\right)$ & 2 & 1 & 3 & $3^{--}$ & 1.8798 & - & $\approx 0$ & \\
\hline \multirow[t]{4}{*}{$5 \mathrm{D}$} & $\eta_{c 2}\left(5^{1} D_{2}\right)$ & 2 & 0 & 2 & $2^{-+}$ & 2.1248 & - & $\approx 0$ & \\
\hline & $\psi\left(5^{3} D_{1}\right)$ & 2 & 1 & 1 & $1^{--}$ & 2.0714 & - & $\approx 0$ & \\
\hline & $\psi_{2}\left(5^{3} D_{2}\right)$ & 2 & 1 & 2 & $2^{--}$ & 2.1218 & - & $\approx 0$ & \\
\hline & $\psi_{3}\left(5^{3} D_{3}\right)$ & 2 & 1 & 3 & $3^{--}$ & 2.1452 & - & $\approx 0$ & \\
\hline \multirow[t]{4}{*}{$1 \mathrm{~F}$} & $h_{c 3}\left(1^{1} F_{3}\right)$ & 3 & 0 & 3 & $3^{+-}$ & 1.0878 & - & $\approx 0$ & \\
\hline & $\chi_{2}\left(1^{3} F_{2}\right)$ & 3 & 1 & 2 & $2^{++}$ & 1.06896 & - & $\approx 0$ & \\
\hline & $\chi_{3}\left(1^{3} F_{3}\right)$ & 3 & 1 & 3 & $3^{++}$ & 1.08628 & - & $\approx 0$ & \\
\hline & $\chi_{4}\left(1^{3} F_{4}\right)$ & 3 & 1 & 4 & $4^{++}$ & 1.09818 & - & $\approx 0$ & \\
\hline \multirow[t]{4}{*}{$2 \mathrm{~F}$} & $h_{c 3}\left(2^{1} F_{3}\right)$ & 3 & 0 & 3 & $3^{+-}$ & 1.4253 & - & $\approx 0$ & \\
\hline & $\chi_{2}\left(2^{3} F_{2}\right)$ & 3 & 1 & 2 & $2^{++}$ & 1.4062 & - & $\approx 0$ & \\
\hline & $\chi_{3}\left(2^{3} F_{3}\right)$ & 3 & 1 & 3 & $3^{++}$ & 1.42387 & - & $\approx 0$ & \\
\hline & $\chi_{4}\left(2^{3} F_{4}\right)$ & 3 & 1 & 4 & $4^{++}$ & 1.43578 & - & $\approx 0$ & \\
\hline \multirow[t]{4}{*}{$3 \mathrm{~F}$} & $h_{c 3}\left(3^{1} F_{3}\right)$ & 3 & 0 & 3 & $3^{+-}$ & 1.725 & - & $\approx 0$ & \\
\hline & $\chi_{2}\left(3^{3} F_{2}\right)$ & 3 & 1 & 2 & $2^{++}$ & 1.7056 & - & $\approx 0$ & \\
\hline & $\chi_{3}\left(3^{3} F_{3}\right)$ & 3 & 1 & 3 & $3^{++}$ & 1.72369 & - & $\approx 0$ & \\
\hline & $\chi_{4}\left(3^{3} F_{4}\right)$ & 3 & 1 & 4 & $4^{++}$ & 1.73579 & - & $\approx 0$ & \\
\hline \multirow[t]{4}{*}{$4 \mathrm{~F}$} & $h_{c 3}\left(4^{1} F_{3}\right)$ & 3 & 0 & 3 & $3^{+-}$ & 2.0 & - & $\approx 0$ & \\
\hline & $\chi_{2}\left(4^{3} F_{2}\right)$ & 3 & 1 & 2 & $2^{++}$ & 1.9802 & - & $\approx 0$ & \\
\hline & $\chi_{3}\left(4^{3} F_{3}\right)$ & 3 & 1 & 3 & $3^{++}$ & 1.9988 & - & $\approx 0$ & \\
\hline & $\chi_{4}\left(4^{3} F_{4}\right)$ & 3 & 1 & 4 & $4^{++}$ & 2.0111 & - & $\approx 0$ & \\
\hline \multirow[t]{3}{*}{$1 \mathrm{G}$} & $\eta_{c 4}\left(1^{1} G_{4}\right)$ & 4 & 0 & 4 & $4^{-+}$ & 1.2589 & - & $\approx 0$ & \\
\hline & $\psi_{3}\left(1^{3} G_{3}\right)$ & 4 & 1 & 3 & $3^{--}$ & 1.25026 & - & $\approx 0$ & \\
\hline & $\begin{array}{l}\psi_{4}\left(1^{3} G_{4}\right) \\
\psi_{5}\left(1^{3} G_{5}\right)\end{array}$ & $\begin{array}{l}4 \\
4\end{array}$ & $\begin{array}{l}1 \\
1\end{array}$ & $\begin{array}{l}4 \\
5\end{array}$ & $\begin{array}{l}4^{--} \\
5^{--}\end{array}$ & $\begin{array}{l}1.25878 \\
1.26409\end{array}$ & (a) & $\begin{array}{l}\approx 0 \\
\approx 0\end{array}$ & \\
\hline
\end{tabular}

Hình 1. Ảnh của tập dữ liệu TabStrucDB và tập dữ liệu DetectCell (a) bảng có cấu trúc các hàng sát nhau của tập dữ liệu TabStrucDB (b) Một cột của bảng đo lưu lượng bằng máy $\mathrm{ADCP}$ tập dữ liệu DetectCell.

Bảng 1. Danh sách các sổ của dữ liệu KTTV.

\begin{tabular}{cllc}
\hline STT & \multicolumn{1}{c}{ Tên sổ } & \multicolumn{1}{c}{ Đặc điểm } & Số mẫu \\
\hline 1 & SKT-1 & Sổ quan trắc khí tượng cơ bản & 642 \\
2 & SKT-2 & Sổ quan trắc khí tượng & 7 \\
3 & SKT-3 & Sổ quan trắc nhiệt độ đất & 7 \\
4 & SKT-13a & Sổ quan trắc bốc hơi GGI-3000 & 7 \\
5 & BKT11 & Tổng lượng bức xạ giờ & 8 \\
6 & BKT11a & Cường độ bức xạ giờ & 8 \\
7 & ADCP & Số ghi chép máy ADCP & 6 \\
8 & Tài liệu chỉnh biên & Tài liệu chỉnh biên & 41 \\
9 & & Sổ dùng khi đo độ sâu & 16 \\
10 & & Sổ ghi quan trắc mực nước & 19 \\
11 & & Sổ ghi đo lưu lượng nước số 1 & 11 \\
12 & & Biểu XL mẫu nước chất lơ lửng & 7 \\
13 & Biểu ghi tốc độ và tính chất lưu lượng triều & 5 \\
\hline Tổng & & & 784 \\
\hline
\end{tabular}


Với dữ liệu ban đầu, có thể phân tập dữ liệu KTTV là những bảng cũ của sổ quan trắc khí tượng có từ năm 1969 đến năm 1985, cũng có những bảng của sổ quan trắc khí tượng cơ bản từ năm 2000 trở về đây. Hình 2 mô tả hai loại bảng trong nhiều loại bảng của tập dữ liệu KTTV. Nghiên cứu cho thấy dữ liệu trong bảng, ngoài là các mực in, còn có các chữ viết tay, dành cho hai loại bảng là lịch sử và hiện đại. Ngoài ra, còn một số bảng kẻ tay cũng được xử lý trong bài báo này. Bước đầu nghiên cứu này sử dụng 130 ảnh đưa vào tập TabStructDB để tiến hành đánh giá thuật toán.

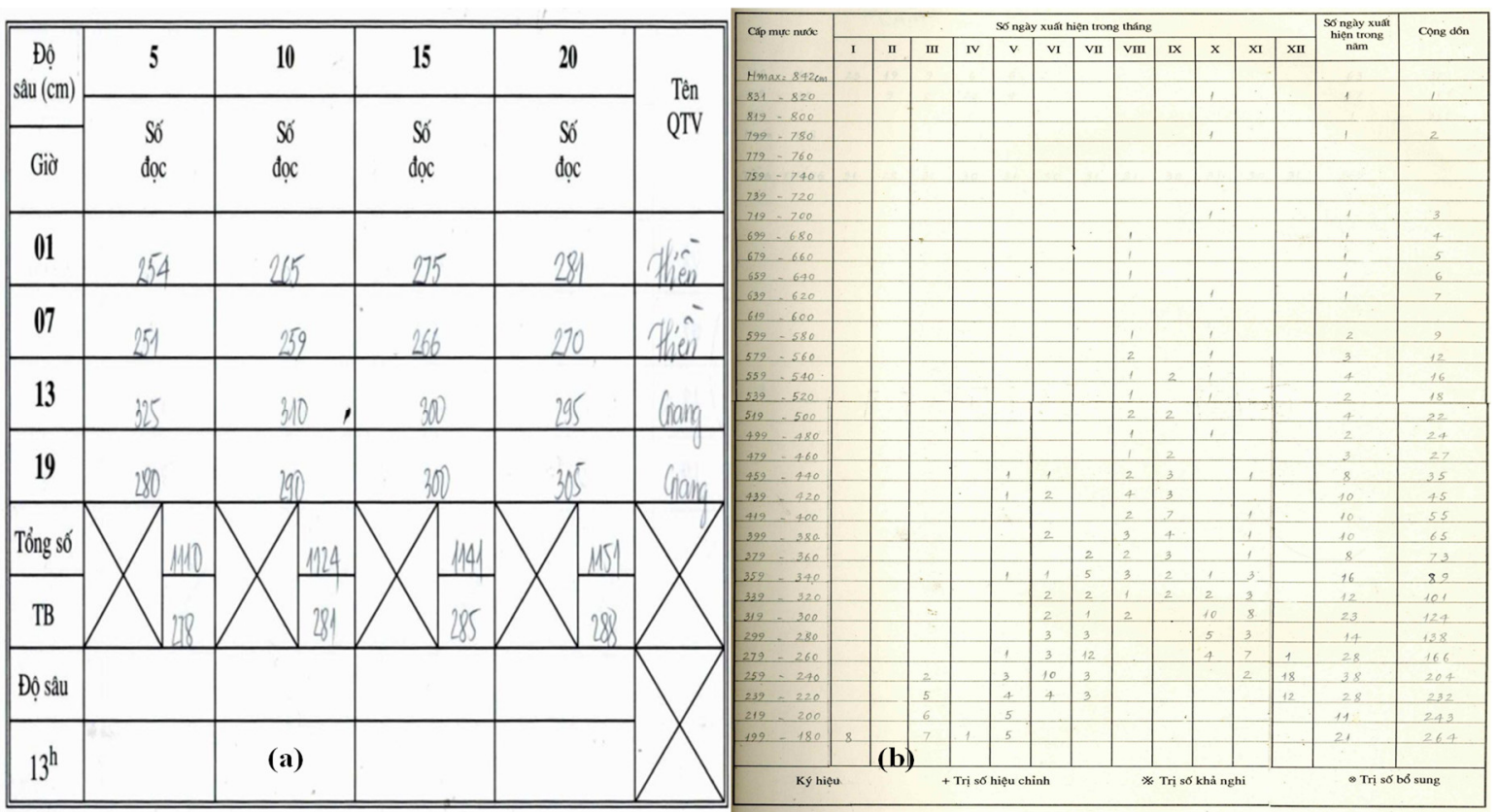

Hình 2. Một số loại bảng thuộc tập dữ liệu Khí tượng thủy văn (a) bảng hiện đại, có các số viết tay (b) bảng lịch sử, có các số viết tay.

\subsection{Phwơng pháp nghiên cứu}

Nghiên cứu sử dụng mô hình Cascade mask r-cnn_r101 fpn dconv c3-c5 để nhận dạng hàng và cột. Do kết quả thực nghiệm ban đầu cho thấy kết quả nhận dạng hàng khá tệ, trong khi kết quả nhận dạng cột tốt. Điều này có thể là do các hàng chứa nhiều phần nền, ít đặc trưng cần thiết để nhận dạng và các hàng có khoảng cách gần nhau. Nghiên cứu này đề xuất phương pháp tiếp tục sử dụng một mạng Faster $\mathrm{R}-\mathrm{CNN}$ dể nhận dạng các cell được tách ra từ các cột vừa được nhận dạng. Ý tưởng này xuất phát từ việc nhận thấy rằng các cell trong một hàng có khả năng dễ dàng được nhận dạng hơn là trong toàn bảng, và các cell nhận dạng trong cột tương đương với một hàng trong bảng. Việc lựa chọn Faster $\mathrm{R}-\mathrm{CNN}$ mục đích muốn lựa chọn một mô hình nhẹ và có hiệu quả cao.

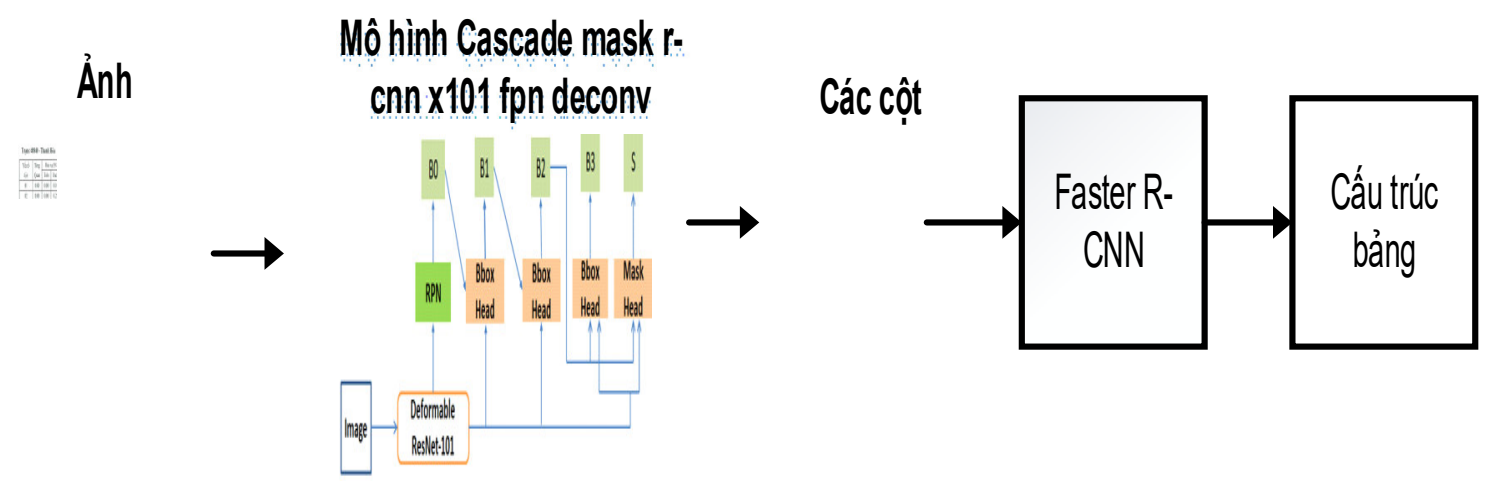

Hình 3. Mô hình nhận dạng cấu trúc bảng. 
Sau đây, sẽ mô tả về một số kỹ thuật được sử dụng trong nhận dạng cấu trúc bảng cụ thể là mô hình Cascade $\mathrm{R}-\mathrm{CNN}$ kết hợp với tích chập biến dạng (Cascade mask $r$-cnn_r101 fpn $d c o n v(3-c 5)$. Mô hình này xuất phát từ một số nghiên cứu trước đây như về $\mathrm{R}-\overline{\mathrm{CNN}}$ [11], Fast R-CNN [12], Faster R-CNN [4], Mask R-CNN [13], Cascade R-CNN [14], tích chập biến dạng [15], ResNet [16], FPN [17]. Mô hình sẽ được huấn luyện trước với tập dữ liệu COCO [18] trước khi được tinh chỉnh lại với dữ liệu của nghiên cứu này. Điểm khác biệt của mô hình này so với các mô hình khác $[3,8]$ là sử dụng tích chập biến đổi. Ta có, phép toán tích chập thông thường như sau:

$$
\left(F^{*} I\right)(i, j)=\sum_{m=-K}^{K} \sum_{n=-K}^{K} F(m, n) \times I(i-m, j-n) \quad \forall i=1, \ldots, H, \forall j=1, \ldots, H
$$

Dấu * thể hiện phép toán tích chập, $\mathrm{F}$ là bộ lọc mà được học, $\mathrm{I}$ là ảnh, $\mathrm{K}$ là giá trị được tính như sau: $[|F| / 2 \mid$ trong đó $|F|$ là kích thước của bộ lọc. $\mathrm{H}$ là chiều cao của ảnh, $\mathrm{W}$ là chiều rộng của ảnh, $\mathrm{i}, \mathrm{j}$ đại diện cho vị trí đang thực hiện phép tích chập đã đề xuất sử dụng phép toán tích chập có thể biến dạng vào trong nhận dạng hàng và cột trong bảng. Phép tích chập biến dạng sử dụng các hệ số mở rộng thay vì sử dụng lưới cố định cho phép lớp tự điều chỉnh. Các hệ số này được tính toán dựa trên tập hợp các tầng tích chập khác, do đó chúng có thể học được. Công thức của phép tích chập biến dạng $2-\mathrm{D}$ có thể được mô tả bằng công thức như sau:

$$
(F \circ I)(i, j)=\sum_{m=-K}^{K} \sum_{n=-K}^{K} F(i, j) \times I\left(i-m+\delta_{i, j, m, n}^{\text {vertical }}, j-n+\delta_{i, j, m, n}^{h \text { orizontal }}\right) \quad \forall \mathrm{i}=1 \ldots \mathrm{H}, \forall j=1 \ldots \mathrm{W}
$$

Kí hiệu $\circ$ thể hiện cho phép tích chập biến dạng, các tham số khác hoàn toàn giống với công thức tích chập thông thường ở trên. $\delta_{i, j, m, n}^{\text {vertical }}, \delta_{i, j, m, n}^{\text {horizontal }}$ kí hiệu cho các tham số mở rộng. Hình 4 thể hiện phép tích chập biến đổi.

Hình 5 các độ lệch (offset) có thể đạt được bằng cách áp dụng một lớp tích chập trên bản đồ đặc trưng đầu vào. Hạt nhân của tích chập sử dụng độ phân giải, độ giãn nở không gian như của lớp tích chập hiện tại. Trường độ lệch (offset) đầu ra có cùng độ phân giải như bản đồ đặc trưng đầu vào và có $2 \mathrm{~N}$ kênh trong đó $2 \mathrm{~N}$ tương ứng với $\mathrm{N}$ hiệu số $2 \mathrm{D}$.

Kết hợp tích chập biến đổi với mạng ResNet-101 [16] và với Cascade Mask $\mathrm{R}-\mathrm{CNN}$ [14], và FPN [17] ta có mô hình mạng như trong Hình 5. Trong đó "I" là ảnh đầu vào, "conv" các tích chập khung xương, "pool" trích rút đặc trưng từng vùng, "H” đầu mạng, "B" hộp giới hạn và "C" là bộ phân loại. "B $\mathrm{B}_{0}$ " là các đề xuất trong toàn bộ kiến trúc.

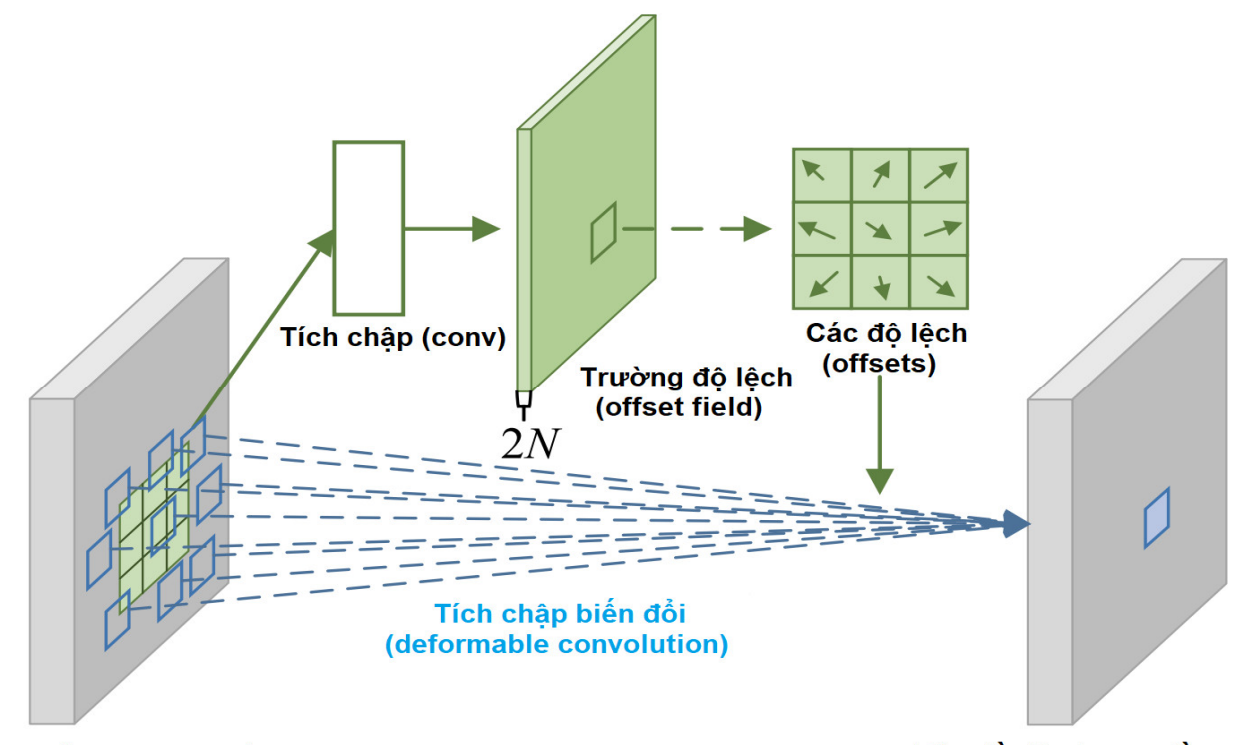

Bản đồ đặc trưng đầu vào

Bản đồ đặc trưng đầu ra

Hình 4. Biểu diễn trực quan của phép tích chập biến đổi. 


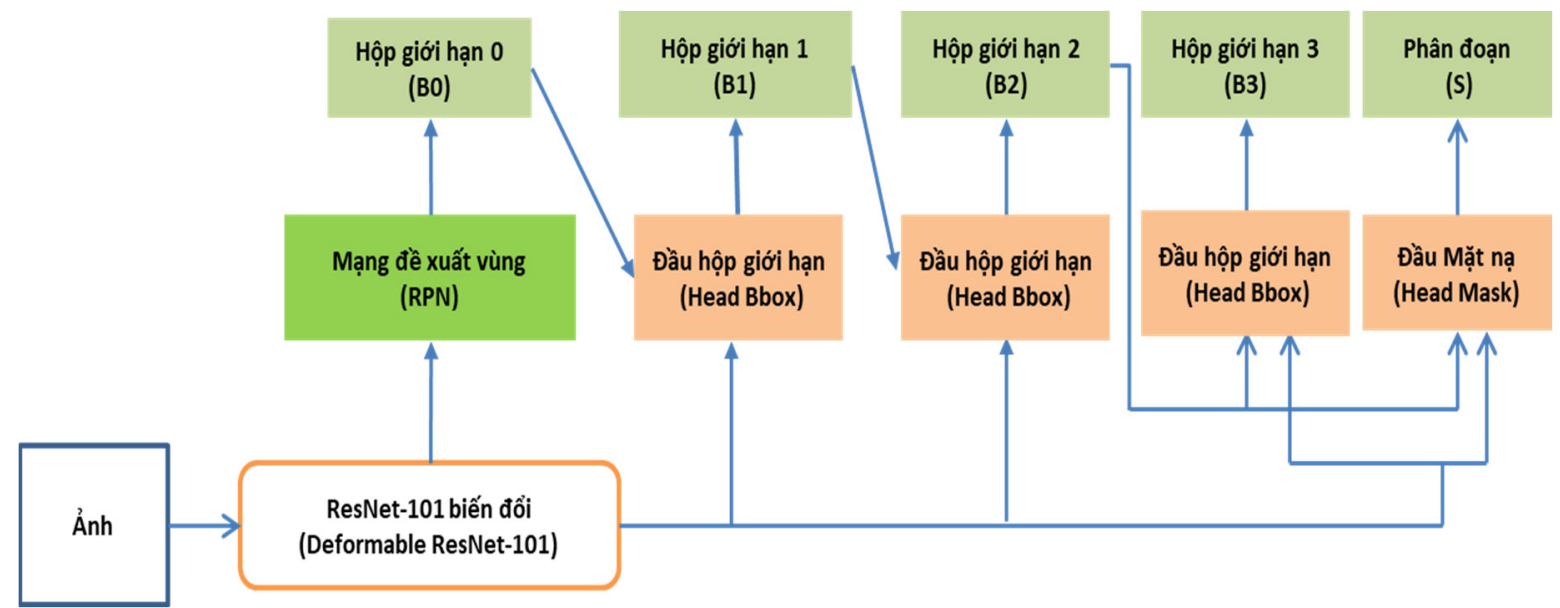

Hình 5. Mô hình mạng Cascade mask r-cnn_r101 dconv.

\section{Kết quả và thảo luận}

\subsection{Tập dũ liệu đánh giá và độ đo}

Nghiên cứu đánh giá trên hai tập dữ liệu TabStructDB mở rộng với 130 bảng thuộc tập dữ liệu KTTV, DetectCell và hiển thị một số kết quả nhận dạng cấu trúc bảng trong tập dữ liệu KTTV. Nghiên cứu này chia các tập dữ liệu theo tỷ lẹ̣ $60 \%$ cho tập huấn luyện, $10 \%$ cho tập kiểm định, $30 \%$ cho tập kiểm tra. Kết quả thực nghiệm được thực hiện trên tập kiểm tra.

Độ đo được sử dụng dựa trên tham số IoU (Intersection over Union) [19] trên các phép đo Precision, Recall, $\mathrm{F} 1$. IoU bằng diện tích vùng chồng lấn giữa hộp dự đoán và hộp đúng (ground truth) chia cho diện tích của vùng được tạo bởi hợp của hộp dự đoán và hộp đúng.

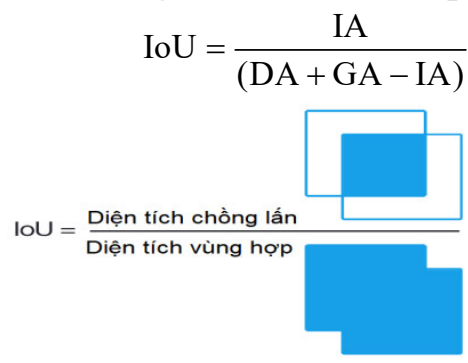

Hình 6. Mô tả về IoU.

Công thức về độ đo $\mathrm{P}, \mathrm{R}, \mathrm{F} 1$ trung bình:

$$
\begin{gathered}
P=\frac{1}{N} \sum_{i=1}^{N}\left(I A_{i} / D A_{i}\right) \\
R=\frac{1}{N} \sum_{i=1}^{N}\left(I A_{i} / G A_{i}\right) \\
F_{1}=2 *\left(\left(P^{*} R\right) /(P+R)\right)
\end{gathered}
$$

Trong đó IA là diện tích vùng giao nhau giữa hộp dự đoán và hộp đúng, DA là diện tích hộp dự đoán, $\mathrm{GA}$ là diện tích hộp đúng, $\mathrm{N}$ là số ảnh trong tập kiểm tra.

\subsection{Kết quả đánh giá}

Nghiên cứu này sử dụng ngôn ngữ lập trình Python, thư viện PyTorch, MMdetection [20] xây dựng chương trình và các mô hình thử nghiệm. Các thực nghiệm của nghiên cứu này được thực hiện trên nền tảng Google Colaboratory Pro với Tesla V100-SXM2 có bộ nhớ GPU 16GB, Intel(R) Xeon(R) CPU @2.30GHz và 24 GB Ram. 


\subsection{1. Đánh giá kết quả nhận dạng hàng và cột}

Thực nghiệm việc nhận dạng cấu trúc bảng. Ta thấy theo như bảng 2 , mô hình cho phép nhận dạng cột khá tốt, tỷ lệ nhận dạng có thể lên tới 97,20\% và độ đo F1 theo IOU lên tới 94,04 \% (độ tin cậy 0,7 ). Tuy nhiên, việc nhận dạng hàng còn chưa tốt chỉ có 49,77\% (độ tin cậy 0,4$)$ hàng có thể nhận ra, những hàng có khả năng nhận ra thì có ngưỡng IoU trùng khớp cao $F 1=89,19 \%$ (độ tin cậy 0,4 ). Các bảng có các hàng cách xa nhau (lớn hơn độ rộng của ký tự) có xu thế nhận dạng rất tốt, các hàng gần nhau thường không thể phân biệt và nhận dạng được. $\mathrm{d}=$ (số đối tượng phát hiện ra có ngưỡng $\mathrm{IoU}>0,5$ )/(số đối tượng thực có).

Bảng 2. Kết quả nhận dạng hàng và cột.

\begin{tabular}{ccccccccccccc}
\hline \multirow{2}{*}{ IoU } & \multicolumn{1}{c}{ Row } & \multicolumn{1}{c}{ Column } & \multicolumn{4}{c}{ All } \\
\cline { 2 - 14 } & $\mathbf{P}$ & $\mathbf{R}$ & $\mathbf{F 1}$ & $\mathbf{d}$ & $\mathbf{P}$ & $\mathbf{R}$ & $\mathbf{F 1}$ & $\mathbf{d}$ & $\mathbf{P}$ & $\mathbf{R}$ & $\mathbf{F 1}$ & $\mathbf{d}$ \\
\hline $\mathbf{0 , 4}$ & 90,72 & 87,71 & $\mathbf{8 9 , 1 9}$ & $\mathbf{4 9 , 7 7}$ & 94,19 & 93,23 & 93,71 & 97,78 & 86,82 & 85,16 & 85,98 & 62,03 \\
$\mathbf{0 , 5}$ & 91,17 & 88,59 & 89,86 & 47,94 & 94,32 & 93,35 & 93,83 & 97,78 & 87,03 & 85,61 & 86,31 & 60,87 \\
$\mathbf{0 , 6}$ & 91,54 & 89,20 & 90,36 & 46,12 & 94,42 & 93,51 & 93,96 & 97,53 & 87,16 & 85,95 & 86,55 & 59,64 \\
$\mathbf{0 , 7}$ & 92,03 & 89,92 & 90,97 & 44,43 & 94,47 & 93,61 & 94,04 & $\mathbf{9 7 , 2 0}$ & 87,34 & 86,27 & 86,80 & 58,53 \\
\hline
\end{tabular}

Nghiên cứu sử dụng một thủ thuật nhỏ là thay đổi kích thước của ảnh theo chiều cao. Nghiên cứu kéo dãn ảnh 3 lần theo chiều cao $\mathrm{h}=\mathrm{h} * 3$, kết quả thực nghiệm cho thấy việc kết hợp kéo dãn ảnh dự đoán hàng và giữ nguyên ảnh gốc cho dự đoán cột cho kết quả tốt nhất như trong bảng 3 . Ta cũng thấy mô hình cũng tốt hơn so với mô hình Cascade mask hrnetv2pw40.

Bảng 3. Kết quả thực nghiệm nhận dạng hàng cột sử dụng kéo dãn ảnh.

\begin{tabular}{|c|c|c|c|c|c|c|}
\hline \multirow{2}{*}{ Phương pháp } & \multirow{2}{*}{ Mô hình } & \multicolumn{4}{|c|}{ IoU } & \multirow{2}{*}{ Wavg. } \\
\hline & & 0,6 & 0,7 & 0,8 & 0,9 & \\
\hline Kéo dãn ảnh & $\begin{array}{l}\text { Cascade mask r-cnn } \\
\text { x101 dconv }\end{array}$ & 83,87 & 84,40 & 85,06 & 85,67 & 84,75 \\
\hline $\begin{array}{l}\text { Ảnh gốc (cột) và } \\
\text { kéo dãn (hàng) }\end{array}$ & $\begin{array}{l}\text { Cascade mask r-cnn } \\
\text { x101 dconv }\end{array}$ & 84,75 & 85,34 & 85,58 & 86,65 & 85,58 \\
\hline $\begin{array}{l}\text { Ảnh gốc (cột) và } \\
\text { kéo dãn (hàng) }\end{array}$ & $\begin{array}{ll}\text { Cascade } & \text { mask } \\
\text { hrnetv2pw40 } & \end{array}$ & 82,53 & 83,55 & 84,52 & 86,08 & 84,17 \\
\hline
\end{tabular}

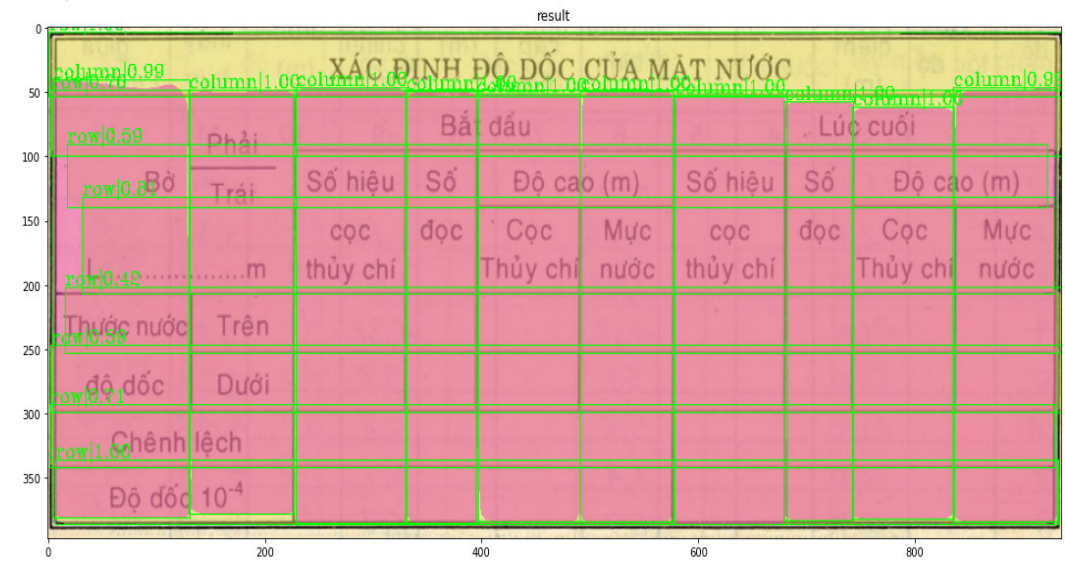

Hình 7. Kết quả nhận dạng hàng cột tốt. 


\begin{tabular}{|c|c|c|c|c|c|c|c|c|c|}
\hline & SkM $\bar{I}^{*}$ & $\beta_{2}$ & $\gamma$ & $\bar{x} x$ & $\overline{\tau_{p}}$ & $\Delta_{n=}$ & $\Delta_{p}$ & $-\lambda_{n i}$ & $-\lambda_{p}$ \\
\hline & 1005in & 0.00 & - & 4.393 & 4.42 & 0.00 & 0.00 & 17.00 & 3.15 \\
\hline & $102 \operatorname{Sin}$ & 0.00 & - & 4.38 & 4.43 & 1.35 & 0.00 & 11.86 & 3.86 \\
\hline & $104 \mathrm{Sn}$ & 0.00 & - & 4.42 & 4.45 & 1.70 & 0.00 & 11.24 & 4.59 \\
\hline & $106 \operatorname{Sin}$ & 0.00 & - & 4.46 & 4.46 & 1.80 & 0.00 & 10.65 & 5.33 \\
\hline & $108 \mathrm{Sin}$ & 0.00 & - & 4.50 & 4.48 & 1.84 & 0.00 & 10.13 & 6.07 \\
\hline 100 & $110 \operatorname{Sin}$ & 0.00 & - & 4.54 & 4.49 & 1.97 & 0.00 & 9.75 & 6.84 \\
\hline & $112 \mathrm{Sin}$ & 0.00 & - & 4.58 & 4.51 & 2.05 & 0.00 & 9.43 & 7.60 \\
\hline & $114 \mathrm{Sn}$ & 0.00 & - & 4.62 & 4.53 & 2.10 & 0.00 & 9.13 & 8.35 \\
\hline 130 & $116 \mathrm{Sin}$ & 0.00 & - & 4.66 & 4.55 & 2.06 & 0.00 & 8.86 & 9.09 \\
\hline & $118 \operatorname{Sin}$ & 0.00 & - & 4.69 & 4.56 & 2.00 & 0.00 & 8.60 & 9.82 \\
\hline & $120 \mathrm{Sin}$ & 0.00 & - & 4.72 & 4.58 & 1.94 & 0.00 & 8.36 & 10.54 \\
\hline 20 & $122 \mathrm{Sn}$ & 0.00 & - & 4.75 & 4.59 & 1.85 & 0.00 & 8.12 & 11.25 \\
\hline & $124 \mathrm{Sn}$ & 0.00 & - & 4.78 & 4.61 & 1.75 & 0.00 & 7.87 & 11.95 \\
\hline & $126 \mathrm{Sin}$ & 0.00 & - & 4.81 & 4.62 & 1.62 & 0.00 & 7.62 & 12.63 \\
\hline & $128 \operatorname{Sin}$ & 0.00 & - & 4.84 & 4.63 & 1.42 & 0.00 & 7.34 & 13.31 \\
\hline & ${ }^{130} \mathrm{Sin}$ & 0.00 & - & 4.87 & 4.64 & 1.10 & 0.00 & 7.01 & 13.98 \\
\hline & $1325 n$ & 0.00 & - & 4.89 & 4.66 & 0.00 & 0.00 & 8.48 & 14.64 \\
\hline & ${ }^{134} \operatorname{Sin}$ & 0.00 & - & 4.93 & 4.67 & 0.68 & 0.00 & 3.62 & 15.07 \\
\hline & $136 \mathrm{Sin}$ & 0.00 & - & 4.97 & 4.68 & 0.78 & 0.00 & 3.36 & 15.51 \\
\hline & 138610 & 500 & - & 5.02 & 4.69 & 1. 04 & 0.00 & 3. 11 & 15.90 \\
\hline & $140 \mathrm{Sin}$ & 0.00 & - & 5.07 & 4.71 & 1.74 & 0.00 & 3.06 & 16.43 \\
\hline
\end{tabular}

Hình 8. Bảng nhận thiếu hàng.

\begin{tabular}{|c|c|c|c|c|c|c|c|c|}
\hline cSkI3 & $\beta_{2}$ & $\bar{\gamma}$ & $\overline{r_{n}}$ & $\overline{\overline{r_{p}}}$ & 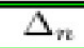 & $\overline{\Delta_{p}}$ & $-\lambda_{n}$ & $-\lambda_{p}$ \\
\hline $3^{100} \mathrm{Sin}$ & 0.00 & - & 4.33 & 4.40 & 0.00 & 0.00 & 17.18 & 3.50 \\
\hline $0^{102} \mathrm{Sn}$ & 0.00 & - & 4.38 & 4.42 & 1.54 & 0.00 & 11.00 & 4.33 \\
\hline $\mathrm{c}^{104} \mathrm{Sn}$ & 0.00 & - & 4.43 & 4.43 & 1.65 & 0.00 & 10.40 & 5.16 \\
\hline $\mathrm{c}^{106} \mathrm{Sn}$ & 0.00 & - & 4.48 & 4.45 & 2.20 & 0.00 & 10.34 & 6.00 \\
\hline$c^{108} \mathrm{Sin}_{11}$ & 0.00 & - & 4.52 & 4.47 & 1.83 & 0.00 & 9.93 & 6.81 \\
\hline$c^{10} \mathrm{Sin}_{11}$ & 0.00 & - & 4.56 & 4.49 & 2.37 & 0.00 & 9.76 & 7.61 \\
\hline d.12 $\mathrm{Sn}$ & 0.00 & - & 4.60 & 4.50 & 2.43 & 0.00 & 9.41 & 8.38 \\
\hline$d^{14} \mathrm{Sn}_{\mathrm{H}}$ & 0.00 & - & 4.64 & 4.52 & 2.31 & 0.00 & 9.09 & 9.13 \\
\hline$c^{116} \mathrm{Sn}$ & 0.00 & - & 4.67 & 4.53 & 2.02 & 0.00 & 8.69 & 9.85 \\
\hline $\mathrm{c}^{18 \mathrm{Sn}}$ & 0.00 & - & 4.71 & 4.55 & 2.04 & 0.00 & 8.28 & 10.55 \\
\hline$c^{120} \mathrm{Sn}$ & 0.00 & - & 4.75 & 4.56 & 0.00 & 0.00 & 8.50 & 10.9 \\
\hline${ }^{122} \mathrm{Sn}$ & 0.00 & - & 4.78 & 4.57 & 1.70 & 0.00 & 7.61 & 11.94 \\
\hline$c^{124} \mathrm{Sn}$ & 0.00 & - & 4.81 & 4.59 & 1.68 & 0.00 & 7.34 & 12.67 \\
\hline $\mathrm{c}^{126} \mathrm{Sn}$ & 0.00 & - & 4.84 & 4.60 & 1.61 & 0.00 & 7.10 & 13.40 \\
\hline $\mathrm{c}^{128} \mathrm{Sn}$ & 0.00 & - & 4.87 & 4.61 & 1.46 & 0.00 & 6.85 & 14.13 \\
\hline $0^{130} \mathrm{Sin}_{11}$ & 0.00 & - & 4.90 & 4.62 & 1.19 & 0.00 & 6.54 & 14.86 \\
\hline $0^{132} \mathrm{Sn}_{11}$ & 0.00 & - & 4.93 & 4.64 & 0.00 & 0.00 & 8.00 & 15.56 \\
\hline $0^{134} \mathrm{Sn}$ & 0.00 & - & 4.99 & 4.65 & 0.78 & 0.00 & 2.00 & 15.93 \\
\hline$c^{136} \mathrm{Sn}$ & 0.00 & - & 5.04 & 4.67 & 1.30 & 0.00 & 2.10 & 16.39 \\
\hline $0^{138} \mathrm{Sn}$ & 0.00 & - & 5.10 & 4.69 & 1.61 & 0.00 & 2.09 & 16.63 \\
\hline${ }^{140} \mathrm{Sn}$ & 0.00 & - & 5.14 & 4.71 & 1.66 & 0.00 & 1.94 & 17.22 \\
\hline
\end{tabular}

Hình 9. Bảng nhận đủ hàng sau khi thực hiện phép kéo dãn.

\subsubsection{Kết quả nhận dạng ô trong bảng}

Sau đó, nghiên cứu này tiến hành thực nghiệm việc nhận dạng ô với từng cột và kết hợp chúng lại với nhau. Bảng 4, thể hiện kết quả nhận dạng ô với tập dữ liệu là các cột.

Bảng 4. Kết quả nhận dạng cell.

\begin{tabular}{|c|c|c|c|c|c|}
\hline \multirow{2}{*}{ Mô hình } & \multicolumn{4}{|c|}{ IoU } & \multirow{2}{*}{ WAvg. } \\
\hline & 0,6 & 0,7 & 0,8 & 0,9 & \\
\hline Faster R-CNN & 88,52 & 88,60 & 88,60 & 88,82 & 88,63 \\
\hline
\end{tabular}


Dưới đây là một số kết quả thực nghiệm nhận dạng cấu trúc bảng từ đầu đến cuối. Qua trình xử lý gồm: phát hiện hàng, cột và phát hiện cell trong bảng. Qua kết quả thực nghiệm, thuật toán có khả năng nhận dạng cấu trúc bảng tương đối tốt. Theo bảng 10 , các ô được nhận dạng chính xác bảng hiện đại, đặc biệt với cả các chữ viết tay. Bảng 11 , bảng hiện đại với các chữ được đánh máy, kết quả nhận dạng các ô chính xác. Bảng 12 , bảng vẽ tay có tính chất lịch sử, chữ viết tay, các ô được nhận dạng chính xác. Bảng 13, bảng lịch sử, giấy có vết gập, và chữ là cả viết tay và đánh máy, thuật toán cho phép ta xác định được chính xác các ô.

\begin{tabular}{|c|c|c|c|c|c|c|c|c|c|c|c|c|c|c|c|}
\hline & Ngay & Gion & Phuit & H & Q & Qhe & $\mathbf{F}$ & Vme & $\mathrm{Vdb}$ & Tèn File so lika tho & Tén Fille Conffg & Nguài do & Huofing chày & Houing do & Ghi Chó \\
\hline 1 & 27 & 17 & .00 & 95 & 282,1 & 282. & 1570 & 0,18 & Q $177^{\circ}$ & $T R 1001$ R. 000 & IR IR A.CFG & Thuit & $=0$ & $I-P$ & Agaid. \\
\hline 2 & 27 & 17 & 10 & & & & & & & $I R T R=01 R \cdot 000$ & IRIRA CFG. & $=$ & $=$ & $P-I$ & \\
\hline 3 & 27 & 17 & 15 & 102. & 363,4 & 36. & 1600 & 0,23 & 0,25 & IRTR $1002 . R .000$ & IRTRA.CFG & $=$ & in: & $T-P$ & $=$ \\
\hline 4 & 27 & 18 & $\infty$. & 120 & 514,6 & $30^{\circ}$ & 1640 & $0,31$. & 0.32 & TRTR 10036.000 & IRIRUCFE. & $+f^{0}$ & A: & $T-p$ & ihe \\
\hline 5 & 27 & 19. & 00 & 140. & 547,9 & sis. & 1690 & 0,32 & 0,32 & IRTR $.007 R .000$ & IRIRA.CFG & Thuat & the & $I-P$ & into \\
\hline 6 & 27 & 20 & $\infty$ & 15.5 & 426,5 & 426 & 1730 & 0,25 & 0,27 & ThIR aro.5R.nt & CRIR A.CFG & This & $=1$ & TP & $=$ \\
\hline 7 & 27 & 20. & 15 & 129 & 426,5 & 426 & 1740. & 0,24 & 626. & IRTR AOOSK Dovo & CRTR AES & $=7$ & $=0$ & $P-T$ & $\frac{160}{-}$ \\
\hline 8 & 27 & 20 & 45. & 106. & 323.5 & 324 & 1760 & 0,18 & 0,16 & IR.1007R.900 & TRIRA.CFG & $=$ & 8 & $T-P$ & $=$ \\
\hline 9. & 27 & 21 & $\infty$ & 169 & 323,6 & 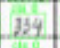 & 6770 & 0.19 & 3, & IS. 1008.8 .700 & TRTRACFE & - & $=$ & $P-T$ & 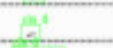 \\
\hline 10 & 27 & 24 & 59 & 173 & 2,6 & 26 & 1780 & 6,0 & 900 & $I R \cdot 1009 \cdot 8000$ & IRIR L OfG & 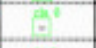 & 30 & $p-T$ & $x_{\| a \hat{z}}$ \\
\hline 11 & 27 & 22 & 55 & 16 & 4587. & 496 & 1760 & 0,26 & 0,32 & IR 100 MaR. $=0$ & TRIK.CFG & $=$ & Me & $A-I$ & II \\
\hline 12 & 27 & 23. & 53 & 152. & 920,4 & 26 & 1720 & 0,53 & $0,60$. & IR. $1041, R .000$ & TRIRLAFE & the & $\frac{10}{4}$ & $P$ & - \\
\hline 13 & 28 & 0 & 53 & 139 & 1059 & joig & 1680 & 0,63 & 0,73 & IR.AO/2.R. $=00$ & IRIENCEG & $=$ & 7. & $8-1$ & $=$ \\
\hline 14 & 28 & 1 & 52 & 119 & 454 & 40 & 1690. & 0,70 & g8s. & IR 1013:R.A20 & TKIRUCFG & $A=0$ & t & $P-1$ & $=0$ \\
\hline 15 & 28 & 2 & 59 & 102 & 1200 & 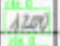 & 4600 & 6,75 & 0,87 & IR $1014 k \cdot 070$ & CRTRL.CFG & 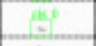 & $t$ & $R-I$ & An! \\
\hline 16 & 28. & 3 & 9 & 87 & 1216 & 1220 & 1560 & 0,78 & 0,89 & $T R 1015 R, 000$ & TEIRACFE. & ila & + & $P-I$ & tel \\
\hline 17 & 48 & 7 & 55 & 14 & 473,5 & 1180 & 1530 & 0,77 & 0,80 & RRAOER .010 & RIR.CFG. & - & + & $p-1$ & $=$ \\
\hline 18 & 28 & 5 & 58. & 63 & usa, & Hies, & 1580 & 0,74 & 0,82 & IR $1017 R \cdot 000$ & RIS. CFA & the & ats & $p-T$ & $\mathrm{Al}^{\circ}$ \\
\hline 19 & 28 & 6. & 56 & & 4332, & 1350 & & & & IR_algR $=00$ & IRIR 1.CPG & Thiy & $7^{\circ}$ & $p-7$ & $=$ \\
\hline 20 & 28 & 7 & 00 & 53 & 143,5 & 4130 & 1480 & 0.76 & 0,83 & $T R_{1020 R}=000$ & TRTRI:CF Q & That & $t^{0}$ & $5-1$ & 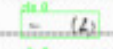 \\
\hline 21 & 28. & 7 & 55 & 47 & 10878 & 109 & 1460 & 0,75 & 0,85 & IR $Q 22 \alpha^{\prime} R, 000$ & TRTR1.OFG & - & + & $p-1$ & tal \\
\hline 22 & 28 & 9 & $\infty$ & 43 & 1094,0 & 1020 & 1410 & 0,75 & 8,10 & $B R \cdot 1022 R=0$ & IRTRICFE & Arupet & \pm & $T-\rho$ & se? \\
\hline 23 & 28. & 9 & & 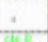 & 10695 & 4020 & & & & $\pi R .1023 R, 006$ & IRTR ICFAG & & $d x$ & $P-I$ & $m$ \\
\hline 24 & 28 & 10 & 100 & 39 & $40028 \mathrm{~A}$ & 1060 & 1440 & 0,74 & 0,81 & CR 10248.000 & $I R T B \cdot C E G$ & ter & + & $7-p$ & \\
\hline
\end{tabular}

Bảng 10. Kết quả nhận dạng cấu trúc bảng kết quả đo lưu lượng bằng máy $\mathrm{ADCP}$.

\begin{tabular}{|c|c|c|c|c|c|c|c|c|c|c|c|c|c|c|c|c|}
\hline \multirow{2}{*}{$\begin{array}{c}\text { Yét to } \\
\text { Gido }\end{array}$} & \multirow{2}{*}{$\begin{array}{l}\text { Tóng } \\
\text { Oann }\end{array}$} & \multicolumn{2}{|c|}{ Buccussy } & \multicolumn{2}{|c|}{$B O E \& 5 D$} & \multirow[b]{2}{*}{ Tann } & \multirow[b]{2}{*}{ Truc xa } & \multirow{2}{*}{$\begin{array}{c}\text { True xa } \\
\text { ngang }\end{array}$} & \multirow[b]{2}{*}{$\operatorname{Tang} \times x_{2}$} & \multirow[b]{2}{*}{ Nhict dof } & \multicolumn{2}{|c|}{ Buic sed the } & \multicolumn{2}{|l|}{ Cin cin } & \multirow{2}{*}{\begin{tabular}{|c|} 
Ther gian \\
ning
\end{tabular}} & \multirow[b]{2}{*}{ Ghi che } \\
\hline & & Trin & Duôi & Trén & Devii & & & & & & $5 \mathrm{~N}$ & SD & búc $x$ & Anbedo & & \\
\hline (0) & 600 & 0.00 & 0.15 & 148.49 & 148.68 & 000 & 0.00 & 0.00 & 143.49 & 18.90 & 0.15 & .019 & -0.33 & 000 & & \\
\hline 02. & 000 & 0.00 & 0.13 & 148.70 & 148.96 & 000 & 0.00 & 00 & 14870 & 1900 & -0.13 & -016 & -0.29 & 000 & & \\
\hline 03 & 600 & 0.00 & 0.05 & 14855 & 148.65 & 000 & 0.00 & 600 & 14855 & 18.93 & -0.05 & .013 & -0.18 & 000 & & \\
\hline 04 & 000 & 0.00 & 0.01 & 14850 & 148.65 & 000 & 0.00 & 00 & 14850 & 1890 & -0.01 & 0.15 & -0.16 & 000 & & \\
\hline 05 & 000 & 0.00 & 0.00 & 14833 & 148.44 & 000 & 0.00 & 0.00 & 148.33 & 18.82 & 0.00 & - 111 & -0.11 & 000 & & \\
\hline 06 & 000 & 0.00 & 0.00 & 14832 & 148.45 & 000 & 0.00 & $\theta \omega$ & 14832 & 18.81 & 0.00 & -0.13 & -0.14 & 0.00 & & \\
\hline 07 & 021 & 0.51 & 0.23 & 148.10 & 148.43 & 026 & 0.00 & 0.00 & 148.42 & 18.71 & 0.08 & .033 & -0.25 & 075 & & \\
\hline 08 & 793 & 8.55 & 1.48 & 14827 & 149.15 & 555 & 764 & 238 & 15682 & 18.79 & 707 & .087 & 6.20 & 0.17 & & \\
\hline 09 & 4248 & 4000 & 7.22 & 15.72 & 152.48 & 28.64 & 28.12 & 13.83 & 194.81 & 19.99 & 36.87 & -1.76 & 35,11 & 0.16 & & \\
\hline 10 & 53.15 & 54.65 & 7.91 & 15466 & 155,20 & 3608 & 2797 & 1707 & 20031 & 2189 & 46.74 & -054 & 4620 & 0.14 & & \\
\hline II & 5330 & 60.16 & 8.86 & 1482 & 155.72 & 39.75 & 2583 & 1854 & 21498 & 21.96 & 51.30 & .090 & 50.40 & 0.15 & & \\
\hline 12 & 50.47 & 5208 & 7.26 & 15600 & 156.33 & 3461 & 2121 & 1586 & 20808 & 2252 & $448 ?$ & -0.33 & 449 & 0.14 & & \\
\hline 13 & 3476 & 3608 & 4.93 & 14.19 & 15462 & 2364 & 15.49 & 11.12 & 190.27 & 21.66 & 31.15 & 043 & 30.72 & 0.14 & & \\
\hline 14 & 41.86 & 42,93 & 6.53 & 1499 & 155.90 & 2853 & 21.14 & 1333 & 197.02 & 2161 & 36,40 & -0.91 & 3549 & 0.15 & & \\
\hline 15 & 2843 & 2965 & 4.36 & 15350 & 154,15 & 1928 & 18.59 & 915 & 183.15 & 21,33 & 25.92 & .065 & 2465 & 0.15 & & \\
\hline 16 & 2994 & 31.03 & 4.84 & 15345 & 154.12 & 20.49 & 30.39 & 945 & 184.48 & 21.31 & 26.18 & 0.67 & 2551 & 0.16 & & \\
\hline 17. & 19.06 & 19.99 & 2.89 & 15305 & 153.36 & 1303 & 59.98 & 603 & 17304 & 2111 & 17.10 & -031 & 16.99 & 0.14 & & \\
\hline 18 & 163 & 1.94 & 0.19 & 151.75 & 151.80 & 126 & .296 & 0.37 & 153.70 & 20.49 & 1.76 & .004 & 1.71 & 0.10 & & \\
\hline 19 & 000 & 0.00 & 0.07 & 15067 & 150.9 & 000 & 0.00 & 000 & 15067 & 1996 & -0.07 & -032 & -0.40 & 0.00 & & \\
\hline 20 & 000 & 0.00 & 0.14 & 15035 & 150.72 & 0.00 & 0.00 & 0.00 & 150.35 & 19.81 & -0.14 & -0.37 & -0.50 & 0.00 & & \\
\hline 21 & 000 & 000 & 0.13 & $150 \%$ & 150,4 & $0 \dot{0}$ & 0.00 & 000 & 15006 & 19.67 & 0.13 & (6) 018 & -0.51 & 0.00 & & \\
\hline 22 & 000 & 0.00 & 0.09 & 149.74 & 150.22 & 0.00 & 0.00 & 0.00 & 14974 & 1951 & -0.09 & -0.48 & -0.57 & 0.00 & & \\
\hline 23 & 000 & 0.00 & 0.12 & 14939 & 14987 & 60 & 0.00 & 0.00 & 14939 & 1934 & -0.12 & -0.48 & 0.60 & 0.00 & & \\
\hline 24 & 000 & 0.00 & 0.14 & 149.26 & 149,76 & 0.00 & 0.00 & 0.00 & 14926 & 1928 & 0.14 & -0.50 & -0.63 & 0.00 & & \\
\hline
\end{tabular}

Bảng 11. Kết quả nhận dạng cấu trúc bảng đo bức xạ giờ. 


\begin{tabular}{|c|c|c|c|c|c|c|c|}
\hline sิt & $H$ & 120 & Fira & $V$ TRa & $Q=V_{x} F$ & $10-Q-0$, & $\Delta \omega$ \\
\hline$T-1$ & $\mathrm{~cm}$ & $m^{3} / \mathrm{s}$ & $m^{2}$ & $m / s$ & $m^{3} / \mathrm{s}$ & & $Q$ \\
\hline & 260 & 525 & 615 & 0,85 & 523 & -2 & 0,38 \\
\hline & 280 & 602 & 684 & 0,88 & 602 & (0) & 8 \\
\hline$\cdot$ & 300 & 687 & 752 & 0,9 & 684 & -3 & 0,44 \\
\hline & 320 & 777 & 820 & 0,95 & 779 & 2 & {$[0,26]$} \\
\hline & 340 & 875 & 889 & 0,98 & 871 & 4 & 0,46 \\
\hline & 360 & 975 & 956 & 1,02 & 975 & 묘 & 0 \\
\hline & 380 & 1085 & 1025 & 10.06 & 1086 & [. & $0,0,0$ \\
\hline & 400 & 1200 & 1095 & 2110. & 1204. & {$[4$} & 0,33 \\
\hline & 420 & 1317 & 11662 & 1,13 & 1313 & -4 & 0,30 \\
\hline & 440 & 1455 & 1230 & 21.8 & 1451 & -4 & 0,27 \\
\hline & 260 & 505 & 615 & 6,82 & 504 & $=-1$ & 0,20 \\
\hline & 280 & 555 & 684. & $0,81 \ldots$ & 554 & -1 & 0,20 \\
\hline & 300 & 615 & 752. & 0,82 & 617 & $\sqrt{2}$ & 0,32 \\
\hline & 320. & 685. & 820 & 9.84 & 689 & 4 & 0.58 \\
\hline & 340 & 760 & 889 & 0,85 & 756 & -4 & 0,53 \\
\hline & 360 & 847 & 956 & 088 & 841 & -6 & 0,71 \\
\hline & 380 & 940 & 1025 & 0,92 & 943 & 3 & 032 \\
\hline & 400 & 1040 & 1095 & 0,95 & 1040 & 0 & (c) \\
\hline & 420 & 1150 & 1162 & {$[0,29]$} & 1150 & [0. & 0 \\
\hline & 440 & 1275 & 1230 & 104 & 1279 & 4 & {$[0,31]$} \\
\hline
\end{tabular}

Bảng 12. Kết quả nhận dạng bảng kiểm tra $\mathrm{Q}$ lịch sử viết tay.

\begin{tabular}{|c|c|c|c|c|c|c|c|c|c|c|c|c|}
\hline \multirow[b]{2}{*}{\begin{tabular}{|c|} 
Só \\
hięu \\
thúy \\
trực \\
\end{tabular}} & \multirow{2}{*}{$\begin{array}{l}\text { Khoàng } \\
\text { cách tù̀ } \\
\text { mớc khơi } \\
\text { điềm (m) }\end{array}$} & \multicolumn{3}{|c|}{ DO SÂU (m). } & \multirow{2}{*}{$\begin{array}{c}\text { Góc } \\
\text { chẹch của } \\
\text { dây cáp } \\
\text { (đọ) }\end{array}$} & \multirow[b]{2}{*}{\begin{tabular}{|c|} 
Tri só \\
hiẹu chinh
\end{tabular}} & \multirow[b]{2}{*}{$\begin{array}{c}\text { Độ sâu } \\
\text { đă hię̣u } \\
\text { chình (m) }\end{array}$} & \multirow{2}{*}{$\begin{array}{l}\text { Hiêu } \\
\text { chinh đẻ } \\
\text { quy vè } \\
\text { mực } \\
\text { nước } \\
\text { dùng đẻ } \\
\text { tính }\end{array}$} & \multirow{2}{*}{$\begin{array}{c}\text { Dộ sâu } \\
\text { đã quy về } \\
\text { mực nước } \\
\text { dùng đẻ } \\
\text { tính }\end{array}$} & \multirow{2}{*}{$\begin{array}{l}\text { Do } \\
\text { cao } \\
\text { đáy } \\
\text { song } \\
\text { (m) }\end{array}$} & \multirow[b]{2}{*}{$\begin{array}{l}\text { Dát d } \\
\text { đáy }\end{array}$} & \multirow[b]{2}{*}{ GHI CHÚ } \\
\hline & & I & II & $\begin{array}{l}\text { Trung } \\
\text { bình }\end{array}$ & & & & & & & & \\
\hline 1 & 2 & 3 & 4 & 5 & 6 & 7 & 8 & 9 & 10 & 11 & 12 & 13 \\
\hline 19 & 150 & 0,0 & $8,1$. & 920 & & $\cdot$ & 920 & & & $-8,4$ & & \\
\hline 20 & 14: & $0,2 \ldots$ & Q.2.2. & 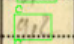 & & & 2,2 & & & 8.6 & & ....... \\
\hline 21 & 130 & 2,1 & 21 & 2.1 & & & 21 & & & -8.5 & & \\
\hline & & & & 6 & 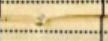 & $\because$ & {$[-\ldots$} & & & & & \\
\hline 22 & $120 \ldots$ & 6... & 6.8 & $6 / 8$ & & & $6,8.8$ & & & $-6,2$ & & \\
\hline 2.3 & 110 & $3.2 ?$ & 3,22 & 3,22 & & & $.3,22$. & & $\therefore$ & 265 & & \\
\hline ALI.LI & 100,6 & $\ldots . . . .$. & ........ & 00 & & & $\infty$ & & & 0.57 & & \\
\hline . & ................. & & & & & & & 5 & & ................ & .......... & \\
\hline $\mathrm{C}_{6}$ & 222.6 & & & & ............. & & & & & $4,78 . \ldots$ & & \\
\hline 65 & 278 & & & $\ldots$ & .............. & & & $\ldots$ & & 1,15 & & \\
\hline C. $4 .$. & 96,0 & 1 & $x$ & $\ldots$ & 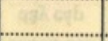 & 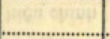 & & 4 & & 151 & ............ & \\
\hline$C_{3}$ & 24,3 & & & & 40 & 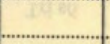 & 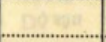 & . & . & 184 & ............. & \\
\hline$c_{2}$ & 9226 & ... & & 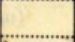 & & & & $x^{2}$ & 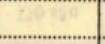 & 214 &.. & \\
\hline G. & 83.8 & & $\cdots$ & & & & & & & 22,2 & & \\
\hline & . & & 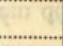 & 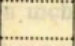 & ……… & $x^{2}$ & & 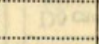 & & & 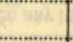 & \\
\hline$k e$ & 00 & ........... & .............. & 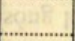 & 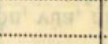 & 1 & & & & 258 & & $\therefore$. \\
\hline & & & 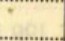 & & & & & & & & & \\
\hline & & & & & & & & & & & & \\
\hline & & & & & & & & & & & & \\
\hline & & & & & & & & & & & & 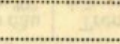 \\
\hline & & & & $(10)$ & (7) & 40 & & & 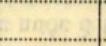 & . & & 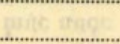 \\
\hline & & & & & & & & & & & & \\
\hline ....... & & & 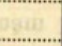 & & 19 & (1) & 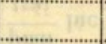 & .......... & 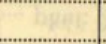 & ……… & & 10 \\
\hline & & & & & & & & & & & & \\
\hline
\end{tabular}

Bảng 13. Kết quả nhận dạng bảng trắc đồ ngang. 


\section{Kết luận}

Nghiên cứu đã đề xuất thuật toán nhận dạng cấu trúc bảng dựa trên mô hình Cascade mask R-CNN x101FPN deconv để nhận dạng hàng và cột, sau đó sử dụng mô hình Faster $\mathrm{R}-$ CNN để nhận dạng các ô trong bảng từ đó đưa ra cấu trúc của bảng. Thuật toán cho thấy hiệu quả cao trong nhận dạng cấu trúc bảng với gần $90 \%$ độ chính xác. Trong thời gian tới, bài báo sẽ tiếp tục nghiên cứu phát triển thuật toán trên thông tin hàng để nâng cao kết quả nhận dạng. Đồng thời tiếp tục thử nghiệm các dữ liệu khác của KTTV để ứng dụng tốt hơn trong thực tế.

Đóng góp của tác giả: Xây dựng ý tưởng nghiên cứu: H.H.P., N.D.D., P.L.P.; Lựa chọn phương pháp nghiên cứu: H.H.P., N.D.D.; Xử lý số liệu: N.D.D.; Phân tích mẫu: N.D.D.; Lấy mẫu: H.H.P., P.L.P.; Viết bản thảo bài báo: H.H.P., N.D.D.; Chỉnh sửa bài báo: H.H.P., N.D.D., P.L.P.

Lời cam đoan: Tập thể tác giả cam đoan bài báo này là công trình nghiên cứu của tập thể tác giả, chưa được công bố ở đâu, không được sao chép từ những nghiên cứu trước đây; không có sự tranh chấp lợi ích trong nhóm tác giả.

\section{Tài liệu tham khảo}

1. Pyreddy, P.; Croft, W.B. Tinti: A system for retrieval in text tables title2. Technical report, USA, 1997.

2. Schreiber, S.; Agne, S.; Wolf, I.; Dengel, A.; Ahmed, S. DeepDeSRT: deep learning for detection and structure recognition of tables in document images. Proceeding of the14th IAPR International Conference on Document Analysis and Recognition (ICDAR), 2017.

3. Ren, S.; He, K.; Girshick, R.; Sun, J. Faster R-CNN: Towards real-time object detection with region proposal networks. IEEE Trans. Pattern Anal. Mach. Intell. 2015, 39, 1137-1149.

4. Dai, J.; Li, Y.; He, K.; Sun, J. R-FCN: Object detection via region-based fully convolutional networks. Proceeding of the $30^{\text {th }}$ Conference on Neural Information Processing Systems (NIPS 2016), Barcelona, Spain, 2016, 379-387.

5. Marmot Dataset. Institute of Computer Science and Techonology of Peking University and Institute of Digital Publishing of Founder R\&D Center, China, http://www.icst.pku.edu.cn/cpdp/data/ marmot_data.htm, 2010.

6. Göbel, M.; Hassan, T.; Oro, E.; Orsi, G. ICDAR 2013 Table Competition. Proceeding of the $12^{\text {th }}$ International Conference on Document Analysis and Recognition, 2013, 1449-1453.

7. Siddiqui, S.A.; Fateh, I.A.; Rizvi, S.T.R.; Dengel, A.; Ahmed, S. DeepTabStR: Deep Learning based Table Structure Recognition. International Conference on Document Analysis and Recognition (ICDAR), 2019, 1403-1409.

8. Prasad, D.; Gadpal, A.; Kapadni, K.; Visave, M.; Sultanpure, K. CascadeTabNet: An approach for end to end table detection and structure recognition from image-based documents. IEEE/CVF Conference on Computer Vision and Pattern Recognition Workshops (CVPRW), 2020.

9. Gao, G.; Huang, Y.; Dejean, H.; Meunier, J.; Yan, Q.; Fang, Y.; Kleber, F.; Lang, E. Icdar 2019 competition on table detection and recognition (ctdar). International Conference on Document Analysis and Recognition (ICDAR), 2019, 1510-1515.

10. Li, M.; Cui, L.; Huang, S.; Wei, F.; Zhou, M.; Li, Z. Tablebank: Table benchmark for image-based table detection and recognition. Proceedings of the $12^{\text {th }}$ Conference on Language Resources and Evaluation, 2020, 1918-1925.

11. Girshick, R.; Donahue, J.; Darrell, T.; Malik, J. Rich feature hierarchies for accurate object detection and semantic segmentation. Proceedings of the IEEE conference on computer vision and pattern recognition, 2014. ISBN:978-1-4799-5118-5. 
12. Girshick, R. Fast R-CNN. Proceedings of the IEEE international conference on computer vision, 2015.

13. He, K.; Gkioxari, G.; Dollar, P.; Girshick, R. Mask r-cnn. ICCV. IEEE 2017, 29802988.

14. Cai, Z.; Vasconcelos, N. Cascade R-CNN: high quality object detection and instance segmentation. IEEE Trans. Pattern Anal. Mach. Intell. 2019, 43, 1483-1498.

15. Dai, J.; Qi, H.; Xiong, Y.; Li, Y.; Zhang, G.; Hu, H.; Wei, Y. Deformable convolutional networks. IEEE International Conference on Computer Vision (ICCV) 2017, 1, pp. 1-3.

16. He, K.; Zhang, X.; Ren, S.; Sun, J. Deep residual learning for image recognition. Proceedings of the IEEE conference on computer vision and pattern recognition, 2016, 770-778.

17. Lin, T.; Dollár, P.; Girshick, R.; He, K.; Hariharan, B.; Belongie, S. Feature Pyramid Networks for Object Detection. IEEE Conference on Computer Vision and Pattern Recognition (CVPR), 2017, 936-944, doi: 10.1109/CVPR.2017.106.

18. Lin, T.Y.; Maire, M.; Belongie, S.; Hays, J.; Perona, P.; Ramanan, D.; Dollár, P.; Zitnick, C.L. Microsoft coco: Common objects in context. In: Fleet D., Pajdla T., Schiele B., Tuytelaars T. (eds) Computer Vision - ECCV 2014. ECCV 2014. Lecture Notes in Computer Science, vol 8693. Springer, Cham. 2014, pp. 740-755. https://doi.org/10.1007/978-3-319-10602-1_48.

19. Rezatofighi, H.; Tsoi, N.; Gwak, J.Y.; Sadeghian, A.; Reid, I.; Savarese, S. Generalized intersection over union: A metric and a loss for bounding box regression. Proceedings of the IEEE/CVF Conference on Computer Vision and Pattern Recognition, 2019, pp. 658-666.

20. Chen, K.; Wang, J.; Pang, J.; Cao, Y.; Xiong, Y.; Li, X.; Sun, S.; Feng, W.; Liu, Z.; Xu, J.; Zhang, Z.; Cheng, D.; Zhu, C.; Cheng, T.; Zhao, Q.; Li, B.; Lu, X.; Zhu, R.; Wu, Y.; Dai, J.; Wang, J.; Shi, J.; Ouyang, W.; Loy, C.C.; Lin, D. Mmdetection: Open MMLab detection toolbox and benchmark. CoRR, abs/1906.07155, 2019.

\title{
Research and apply table structure recognition algorithm based on object detection
}

\author{
Ngo Dai Duong1, Hai-Hong Phan ${ }^{1 *}$, Pham Le Phuong ${ }^{2}$ \\ ${ }^{1}$ Military Technical Academy, Viet Nam; hongpth@1qdtu.edu.vn; \\ daiduong28789@hotmail.com \\ ${ }^{2}$ Hydro-Meteorological Information and Data Center; phuongp180@gmail.com
}

\begin{abstract}
Table structure identification is an important issue in document digitization. With the development of current deep learning techniques, the detection of tables has made great strides, while table structure identification still faces many difficulties due to complex table structure, especially with real data practice. This article proposes a method to apply the Cascade mask model R-CNN x101FPN deconv to identify rows and columns. The paper also proposes to use the Faster R-CNN model to identify the cells in the table, thereby giving out the table structure. The proposed algorithm is evaluated on popular datasets such as TabStructDB and documents collected from Hydrometeorology stations. The experimental results reached $90 \%$ accuracy on these datasets. The algorithm is capable of effectively applying to the identification of the table structure of common documents; especially, the algorithm is capable of dealing with historical documents and handwriting, in accordance with the document characteristics of the hydrometeorology industry. Since then, it contributes to the digitization of documents, storage and data retrieval of hydrometeorology industry.
\end{abstract}

Keywords: Structure table recognition; Cell recognition; Meteorological and hydrological. 